\title{
A Fast Acquisition Algorithm Overcoming Fuzz Problems for TDDM Spread Spectrum Signal
}

\author{
Fang Liu and Yongxin Feng \\ School of Information Science and Engineering, Shenyang Ligong University, Shenyang 110159, China \\ Correspondence should be addressed to Yongxin Feng; onceowned_1019@163.com
}

Received 4 February 2014; Revised 9 April 2014; Accepted 5 May 2014; Published 28 May 2014

Academic Editor: Wang Xing-yuan

Copyright ( 2014 F. Liu and Y. Feng. This is an open access article distributed under the Creative Commons Attribution License, which permits unrestricted use, distribution, and reproduction in any medium, provided the original work is properly cited.

\begin{abstract}
TDDM (time division data modulation) technique will be used in the next generation GNSS (global navigation satellite system) to improve processing performance and to reduce inter-GNSS interference; however, the emergence of TDDM signal causes the estimation frequency and message reversal fuzz problems in the acquisition process of a GNSS receiver. At present, the traditional acquisition methods have some limitations and shortcomings. Therefore, aiming at the unique characteristics of TDDM signal, a fast acquisition algorithm is proposed to overcome these fuzz problems in this paper. In the proposed algorithm, three stages are obtained by some key technologies, which are the $I$ - $Q$ frequency compensation, superposition processing, subsection processing, and reversion position estimation. Besides, the algorithm is simulated from carrier frequency error, code phase error, message inversion error, and processing speed. Theoretical and simulation results show that the new algorithm can quickly overcome the fuzz problems, and the new algorithm is better than the existing algorithm in the speed and accuracy, which demonstrates that this new algorithm is an effective search scheme for the next generation GNSS signals.
\end{abstract}

\section{Introduction}

GNSS (global navigation satellite system) [1] with the advantages of high accuracy, high speed, and low cost has become the most widely used application of spread spectrum communication technology $[2,3]$. At the same time, TDDM (time division data modulation) [4] technique will be used in the next generation GNSS (global navigation satellite system) to improve processing performance and to reduce inter-GNSS interference. The mechanisms of TDDM modulation are a new spread spectrum technology using long-period code parity. Therefore, the TDDM signal has not only the characteristics of long codes but also the unique characteristics of this modulation method.

In the spread spectrum communication technology research, the signal synchronization research plays a more and more important role in a receiver. Only by owning the signal synchronization ability, we can achieve the purpose of expectant measure, investigation, communication, navigation, and so on. So some synchronization acquisition algorithms are proposed, such as the long code acquisition algorithms [5-7] and the relevant algorithms based on FFT
$[8,9]$. At the same time, many fast acquisition algorithms [10-13] are also proposed from the perspective of processing speed, and some improved XFAST algorithms $[14,15]$ have been proposed. Furthermore, some new studies on analysis of the signal synchronization are proposed, such as activation feedback control synchronization scheme [16] and projective synchronization scheme [17]. These algorithms and schemes can reach effective synchronization purpose.

However, considering some synchronization problems, especially acquisition fuzz problems, are caused just because of the TDDM signal comprehensive properties, the above algorithms and schemes cannot solve the acquisition fuzz problems for TDDM signal. At present, the two-channel algorithm [18] is proposed to solve the fuzz problems of TDDM signal. Although the algorithm can deal with fuzz problems, the algorithm precision is not enough, and the processing speed is slow.

In summary, according to the TDDM signal characteristics, combined with the existing research methods, a fast acquisition algorithm is proposed to overcome the fuzz problems for TDDM signal in this paper. 


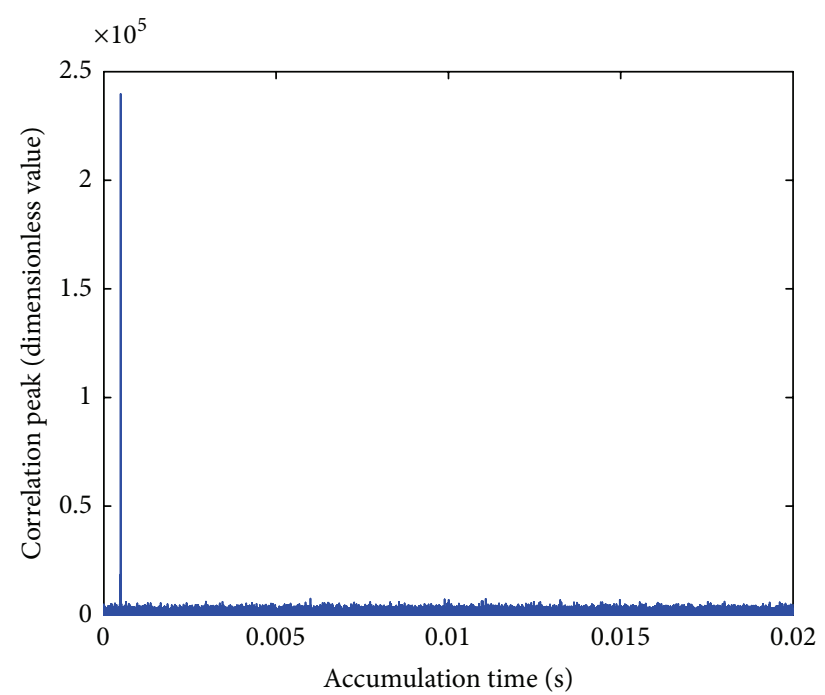

(a) The theoretical correlation result

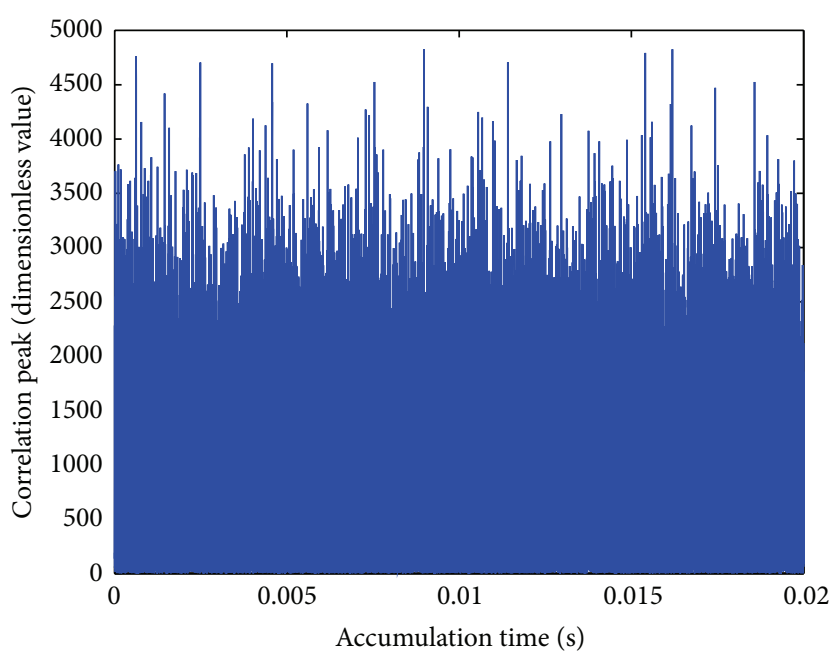

(b) The acquisition result

FIgURE 1: The correlation results in the condition of $20 \mathrm{~ms}$ accumulation time.

\section{The Fuzz Problems of TDDM Signal Acquisition}

2.1. The Fuzz Problem Caused by External Factors. Evident frequency error will appear due to relative motion when the signal arrives at the receiver, so the frequency mixing technology of multiple channels is usually used in traditional acquisition algorithms to overcome this problem. However, the residual frequency exists in the mixing signal, which will affect the correlation result. And if the residual frequency satisfies the following equation, the maximum error [19] will appear in the correlation result. Consider

$$
t \times \Delta f=\frac{1}{2} \times n \quad(n=1,2 \cdots+\infty),
$$

where $t$ is the accumulation time, $\Delta f$ is the residual frequency, and $n$ is a positive integer.

Because the residual frequency is periodic function, the maximum error will appear in the correlation result when the single cycle of residual frequency satisfies the following equation:

$$
f_{e}=\frac{1}{2 t}
$$

where $f_{e}$ is a cycle of the residual frequency.

Now, the effect of residual frequency is verified by an example. In the condition of $20 \mathrm{~ms}$ accumulation time, the theoretical correlation result is shown in Figure 1(a). When the other conditions are the same, but the residual frequency is $25 \mathrm{~Hz}$, the acquisition result was shown in Figure 1(b). These test results show that the correlation result cannot correctly be gotten when the residual frequency satisfies (2). Therefore, the influence of residual frequency is very great which causes fuzz problems of leakage acquisition.
2.2. The Fuzz Problem Caused by Internal Factors. In TDDM spread spectrum processing, the odd code of PN code is modulated by message, but the even code of $\mathrm{PN}$ code is not modulated by message. If the message sign in the received signal is positive and there is no sign inversion, the correct acquisition result can be gotten in the traditional methods. However, if the message sign in the received signal is negative and there is no sign inversion, the test result of traditional acquisition method is shown in Figure 2. If the message sign in the received signal is not only positive but also negative, the test result of traditional acquisition method is shown in Figure 3. These test results showed that the TDDM mechanism caused the acquisition fuzz problems.

\section{The Fast Acquisition Algorithm Overcoming Fuzz Problems}

Currently, the two-channel algorithm [19] is an effective algorithm for TDDM signal. Firstly, the local code is divided into two channels in the two-channel algorithm. Then, circular correlation operations are completed between each channel signal and received signal of frequency compensation. Finally, each channel maximum peak is calculated, and the message reversion position is calculated. In the process of acquisition, the two-channel algorithm is suitable for the TDDM signal without message inversion, while it is not suitable for the TDDM signal with message inversion. Therefore, this algorithm cannot solve well the fuzz problems caused by TDDM modulation mechanism, and it cannot solve the fuzz problems caused by frequency compensation because the residual frequency influence has not been considered.

Through the above analysis, we can see that the fuzz problems are caused by residual frequency and TDDM mechanism in the process of TDDM signal acquisition. At the same time, the existing algorithm has the limitations 


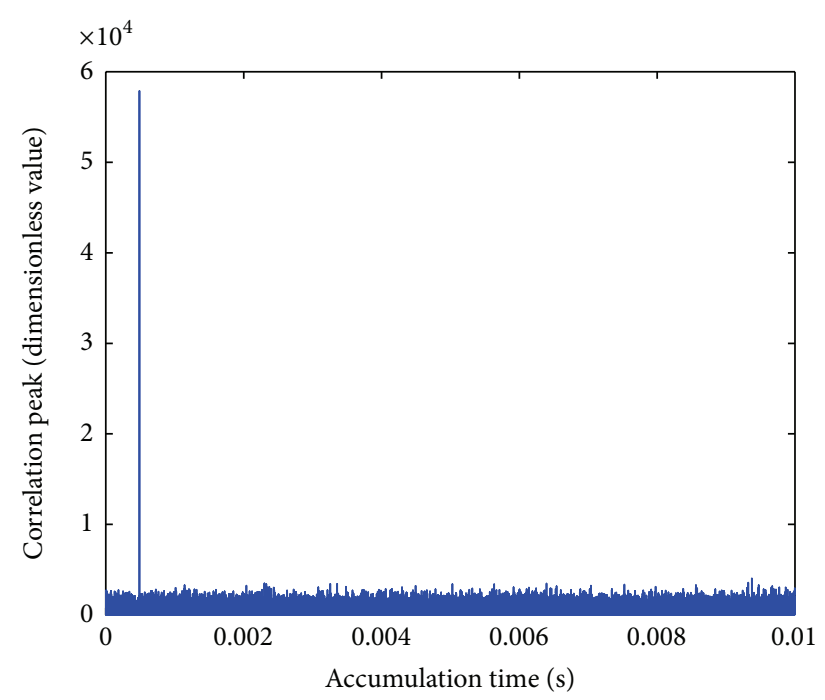

(a) The theoretical correlation result

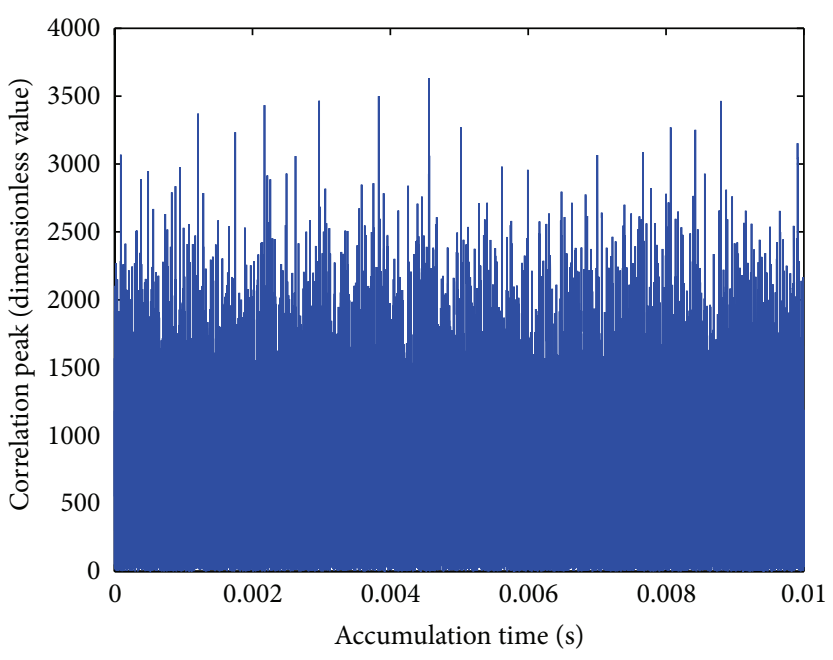

(b) The acquisition result

FIGURE 2: The test results in the condition of negative message sign.

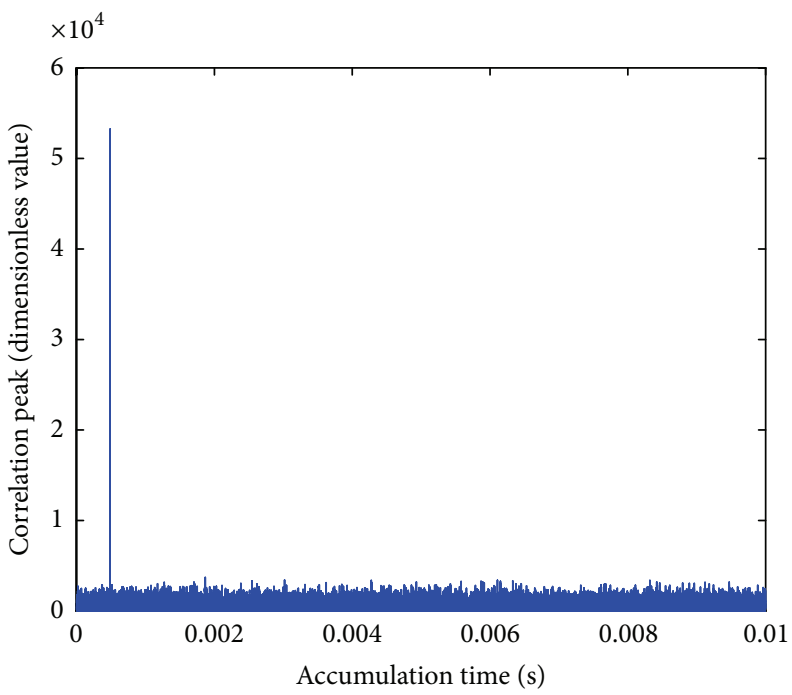

(a) The theoretical correlation result

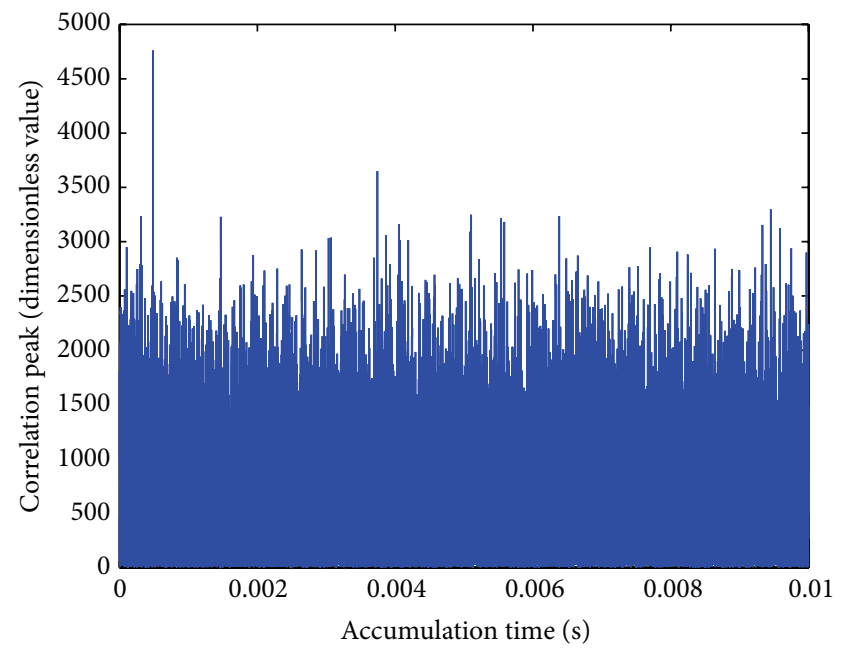

(b) The acquisition result

FIGURE 3: The test results in the condition of positive and negative message sign.

of leakage acquisition and erroneous acquisition. Therefore, a fast acquisition algorithm overcoming fuzz problems is proposed, aiming at the unique characteristics of TDDM signal.

3.1. General Scheme. The new algorithm mainly includes search stage, coarse acquisition stage, and precise acquisition stage, and this algorithm general scheme is shown in Figure 4.

In the search stage, the search speed is the key problem because of the bigger error, so the I-Q frequency compensation processing is employed to overcome the frequency fuzz problem; the superposition processing of local code square wave modulation and original code is employed to overcome message reversal fuzz problem. Further, the search speed is enhanced by subsection processing.

In the coarse acquisition stage, the signal phase and frequency error are quickly determined by removing subsection processing and further $I-Q$ frequency compensation processing.

In the precise acquisition stage, the received signal and local code are divided into two sections, and the length of each section is equal to the duration of half message code. If there is sign reversion in one section of received signal, there is surely no sign reversion in another section. 


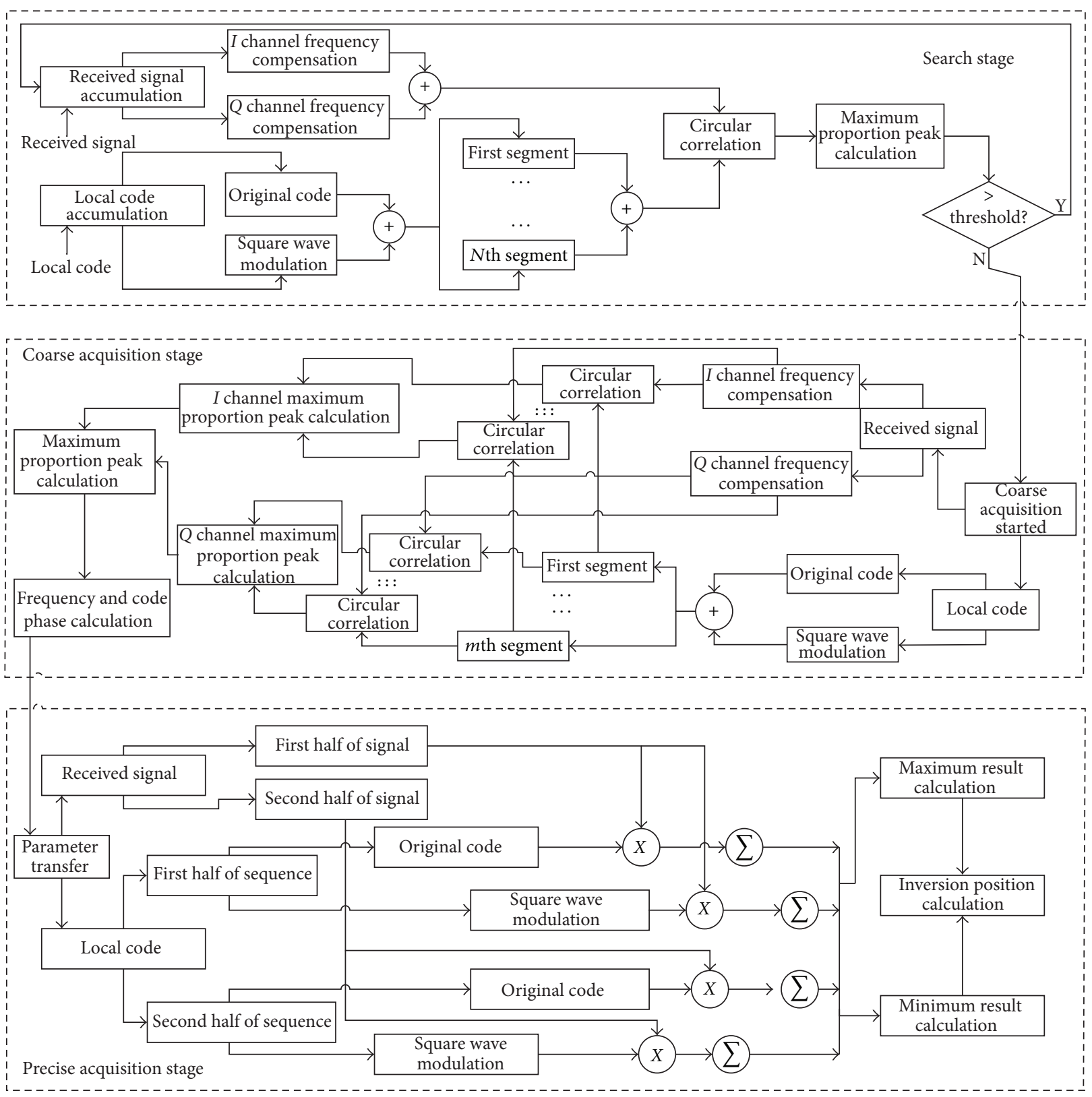

FIGURE 4: The general scheme of the fast acquisition algorithm.

So the technology is used to find reversion section and combined with four channel correlation results to calculate message reversion position.

3.2. Algorithm Principle. The TDDM spread spectrum process is shown in (3), where $d(n)$ is the message code, $C(n)$ is the PN code, $S N\left(\omega_{S} n\right)$ is the square wave, and $\omega_{S}$ is the frequency of square wave. If the message sign is positive in accumulation time, the TDDM spread spectrum result is equivalent to the product of message code and PN code. If the message sign is negative, the TDDM spread spectrum result is multiplied by the square wave. Further, the received signal is shown in (4), where $\omega_{R}$ is the intermediate frequency and $\varphi_{R}$ is the carrier phase. Consider

$$
\begin{aligned}
C_{\mathrm{TDDM}}(n) & =\left.(d(n) C(n))\right|_{T} \\
& = \begin{cases}d(n) C(n), & d(n)=1 \\
d(n) C(n) S N\left(\omega_{S} n\right), & d(n)=-1\end{cases} \\
S_{R}(n) & =A C_{\mathrm{TDDM}}(n) \cos \left(\omega_{R} n+\varphi_{R}\right) \\
& =\left.A(d(n) C(n))\right|_{T} \cos \left(\omega_{R} n+\varphi_{R}\right) .
\end{aligned}
$$


Firstly, the received signal is transmitted into the search stage. In this stage, the parallel $I-Q$ frequency compensation processes are employed, and the processes are expressed as

$$
\begin{aligned}
S_{I}(n) & =S_{R}(n) \cos \left(\omega_{b} n+\phi_{b}\right) \\
& =\left.A(d(n) C(n))\right|_{T} \cos \left(\Delta \omega_{I} n+\Delta \varphi\right) \\
S_{Q}(n) & =S_{R}(n) \cos \left(\left(\omega_{b}+\frac{\omega_{e}}{2}\right) n+\varphi_{b}\right) \\
& =\left.A(d(n) C(n))\right|_{T} \cos \left(\Delta \omega_{\mathrm{Q}} n+\Delta \varphi\right),
\end{aligned}
$$

where $\omega_{b}$ is the basal frequency, $\varphi_{b}$ is the basal carrier phase, $\omega_{e}$ is the residual frequency, $\Delta \varphi$ is the phase after frequency compensation, and $\Delta \omega_{I}$ and $\Delta \omega_{Q}$ are the frequency of $I$ channel and $Q$ channel, respectively.

At the same time, $m$ segments local codes are accumulated; each segment length is equal to the length of the received signal, and then the local original code and its square wave modulation results are added, and the process is expressed as

$$
\begin{aligned}
G_{i}(n)= & C_{Y}((i-1) N+n) \\
& +C_{Y}((i-1) N+n) S N\left(\omega_{S} n\right), \quad(i=1,2 \cdots m),
\end{aligned}
$$

where $N$ is the point number of correlation processing.

Each segment superposition result is expressed as

$$
\begin{aligned}
& G_{X}(n)= \sum_{i=1}^{m} G_{i}(n) \\
&=\sum_{i=1}^{m} C_{Y}((i-1) N+n) \\
& \quad \quad+C_{Y}((i-1) N+n) S N\left(\omega_{S} n\right) .
\end{aligned}
$$

Then, the circular correlation operation is completed by using the results of (5) and (7), and the process is expressed as

$$
\begin{aligned}
& X(n)= {\left[\sum_{m=0}^{N}\left(S_{I}(m)+S_{Q}(m)\right) G_{X}^{*}((m-n))_{N}\right] R_{N}(n) } \\
&= {\left[\sum_{m=0}^{N} S_{I}(m) G_{X}^{*}((m-n))_{N}+S_{Q}(m) G_{X}^{*}((m-n))_{N}\right] } \\
& \times R_{N}(n) \\
&= {\left[\sum _ { m = 0 } ^ { N } \left\{S_{I}(m)\right.\right.} \\
& \times\left(\sum_{i=1}^{m} C_{Y}^{*}(((i-1) N+m-n))_{N}\right.
\end{aligned}
$$

$$
\begin{gathered}
+C_{Y}^{*}(((i-1) N+m-n))_{N} \\
\left.\times S N\left(\left(\omega_{S}(m-n)\right)\right)_{N}\right) \\
+S_{Q}(m)\left(\sum_{i=1}^{m} C_{Y}^{*}(((i-1) N+m-n))_{N}\right. \\
+C_{Y}^{*}(((i-1) N+m-n))_{N} \\
\left.\left.\left.\times S N\left(\left(\omega_{S}(m-n)\right)\right)_{N}\right)\right\}\right]
\end{gathered}
$$$$
\times R_{N}(n)
$$$$
\approx \sum_{m=0}^{N} A d(m) \cos \left(\Delta \omega_{\min } m+\Delta \varphi\right)
$$$$
+\sum_{j=1}^{4 m-1} \sum_{m=0}^{N} A d(m) C(m) D_{j}(m)
$$$$
\times \cos \left(\Delta \omega_{\text {other }} m+\Delta \varphi\right)
$$$$
\approx \sum_{m=0}^{N} A d(m) \cos \left(\Delta \omega_{\min } m+\Delta \varphi\right)+\sum_{j=1}^{4 m-1} \lambda_{j},
$$

where $R_{N}(n)$ is the rectangle sequence, $G_{X}^{*}((m-n))_{N}$ is the result of conjugate and circular shifting for $G_{X}(n)$, and $D_{j}(m)$ is the other PN code.

Because there is no correlation between $D_{j}(m)$ and the received signal, the correlation result of $D_{j}(m)$ and the received signal is equivalent to noise, and the noise is expressed as $\lambda_{i}$. The maximum proportion peak is calculated using the correlation results, and the process is expressed as

$$
P_{V}=\frac{N \cdot \max [X(n)]}{\sum_{n=1}^{N}[X(n)]}
$$

The frontal operations are redone using new received signal if $P_{V}$ cannot meet the threshold; otherwise, the received signal is transmitted in the coarse acquisition stage.

In the coarse acquisition stage, the estimated frequency value $\omega_{d}$ is calculated by using the channel of $P_{V}$, and the $I-Q$ frequency compensation processes for received signal are done anew; they are expressed as

$$
\begin{aligned}
S_{I}^{\prime}(n) & =S_{R}(n) \cos \left(\omega_{d} n+\phi_{d}\right) \\
& =\left.A(d(n) C(n))\right|_{T} \cos \left(\Delta \omega_{I}^{\prime} n+\Delta \varphi\right) \\
S_{Q}^{\prime}(n) & =S_{R}(n) \cos \left(\left(\omega_{d}+\frac{\omega_{e}}{2}\right) n+\varphi_{d}\right) \\
& =\left.A(d(n) C(n))\right|_{T} \cos \left(\Delta \omega_{\mathrm{Q}}^{\prime} n+\Delta \varphi\right) .
\end{aligned}
$$


The circular correlation between $S_{I}^{\prime}(n), S_{\mathrm{Q}}^{\prime}(n)$, and $G_{i}^{*}$ $((m-n))_{N}$ is expressed as

$$
\begin{aligned}
& X_{I i}(n)=\left[\sum_{m=0}^{N} S_{I}^{\prime}(m) G_{i}^{*}((m-n))_{N}\right] R_{N}(n) \\
& =\left[\sum _ { m = 0 } ^ { N } \left\{S _ { I } ^ { \prime } ( m ) \left(C_{Y}(((i-1) N+m-n))_{N}\right.\right.\right. \\
& +C_{Y}(((i-1) N+m-n))_{N} \\
& \left.\left.\left.\times S N\left(\left(\omega_{S}(m-n)\right)\right)_{N}\right)\right\}\right] R_{N}(n) \\
& \approx \sum_{m=0}^{N}\left(A d(m) \cos \left(\Delta \omega_{I}^{\prime} m+\Delta \varphi\right)\right) \\
& +\sum_{j=1}^{4 m-1} \sum_{m=0}^{N}\left(A d(m) C(m) D_{j}(m)\right. \\
& \left.\times \cos \left(\Delta \omega_{I}^{\prime} m+\Delta \varphi\right)\right) \\
& \approx \sum_{m=0}^{N} A d(m) \cos \left(\Delta \omega_{I}^{\prime} m+\Delta \varphi\right)+\sum_{j=1}^{4 m-1} \lambda_{j} \\
& X_{\mathrm{Q} i}(n)=\left[\sum_{m=0}^{N} S_{\mathrm{Q}}^{\prime}(m) G_{i}^{*}((m-n))_{N}\right] R_{N}(n) \\
& =\left[\sum _ { m = 0 } ^ { N } \left\{S _ { Q } ^ { \prime } ( m ) \left(C_{Y}(((i-1) N+m-n))_{N}\right.\right.\right. \\
& +C_{Y}(((i-1) N+m-n))_{N} \\
& \left.\left.\left.\times S N\left(\left(\omega_{S}(m-n)\right)\right)_{N}\right)\right\}\right] R_{N}(n) \\
& \approx \sum_{m=0}^{N}\left(A d(m) \cos \left(\Delta \omega_{\mathrm{Q}}^{\prime} m+\Delta \varphi\right)\right) \\
& +\sum_{j=1}^{4 m-1} \sum_{m=0}^{N}\left(A d(m) C(m) D_{j}(m)\right. \\
& \left.\times \cos \left(\Delta \omega_{\mathrm{Q}}^{\prime} m+\Delta \varphi\right)\right) \\
& \approx \sum_{m=0}^{N} A d(m) \cos \left(\Delta \omega_{\mathrm{Q}}^{\prime} m+\Delta \varphi\right)+\sum_{j=1}^{4 m-1} \lambda_{j} .
\end{aligned}
$$

Further, the maximum proportion peaks are calculated using the above results, as (12). The final estimated frequency is calculated using the channel number of $P_{X i}$, and the code phase is calculated using the position of $P_{X i}$. Through the above operations, the carrier frequency and code phase can be controlled in a certain range. However, the message inversion fuzz problem still exists, so the received signal is transmitted into the precise acquisition stage to estimate message inversion position. Consider

$$
\begin{aligned}
P_{I i} & =\frac{N \cdot \max \left[X_{I i}(n)\right]}{\sum_{n=1}^{N}\left[X_{I i}(n)\right]} \\
P_{\mathrm{Q} i} & =\frac{N \cdot \max \left[X_{\mathrm{Q} i}(n)\right]}{\sum_{n=1}^{N}\left[X_{\mathrm{Q} i}(n)\right]} \\
P_{X i} & =\max \left\{\max \left[P_{I i}\right], \max \left[P_{\mathrm{Q} i}\right]\right\} .
\end{aligned}
$$

In the acquisition stage, the received signal is divided into two sections, in which each section's length is equal to the duration of half message code. The frequency compensation of each section is processed by using the result of coarse acquisition stage, and the processes are expressed as

$$
\begin{array}{r}
S_{1}(n)=\left.A(d(n) C(n))\right|_{T} \cos \left(\omega_{R} n+\varphi_{R}\right) \cos \left(\omega_{g} n+\varphi_{g}\right) \\
=\left.A(d(n) C(n))\right|_{T} \cos \left(\Delta \omega_{g} n+\Delta \varphi_{g}\right), \\
\quad(n=1,2 \cdots L), \\
S_{2}(n)=\left.A(d(n) C(n))\right|_{T} \cos \left(\omega_{R} n+\varphi_{R}\right) \cos \left(\omega_{g} n+\varphi_{g}\right) \\
=\left.A(d(n) C(n))\right|_{T} \cos \left(\Delta \omega_{g} n+\Delta \varphi_{g}\right), \\
\quad(n=L+1, \cdots 2 L),
\end{array}
$$

where $\Delta \omega_{g}$ is the frequency error and $L$ is the sampling point.

Then, the local code is generated anew according to the result of coarse acquisition stage, and it is also divided into two sections. The first half of sequence is expressed as in (14) and the second half of sequence is expressed as in (15). Consider

$$
\begin{aligned}
& G_{1}(n)=C_{Y}(n), \quad(n=1,2 \cdots L) \\
& G_{1}^{\prime}(n)=C_{Y}(n) S N\left(\omega_{S} n\right), \quad(n=1,2 \cdots L) \\
& G_{2}(n)=C_{Y}(n), \quad(n=L+1, L+2 \cdots 2 L) \\
& G_{2}^{\prime}(n)=C_{Y}(n) S N\left(\omega_{S} n\right), \quad(n=L+1, L+2 \cdots 2 L) .
\end{aligned}
$$

Furthermore, the correlation process is expressed as

$$
\begin{gathered}
X_{1}=\sum_{n-1}^{L} G_{1}(n) S_{1}(n) \\
X_{1}^{\prime}=\sum_{n-1}^{L} G_{1}^{\prime}(n) S_{1}(n) \\
X_{2}=\sum_{n=L+1}^{2 L} G_{2}(n) S_{2}(n) \\
X_{2}^{\prime}=\sum_{n=L+1}^{2 L} G_{2}^{\prime}(n) S_{2}(n) .
\end{gathered}
$$


The maximum of four correlation results is expressed as in (17), and the minimum is expressed as in (18). Consider

$$
\begin{aligned}
X_{\max } & =\max \left[X_{1}, X_{1}^{\prime}, X_{2}, X_{2}^{\prime}\right] \\
X_{\min } & =\max \left[X_{1}, X_{1}^{\prime}, X_{2}, X_{2}^{\prime}\right] .
\end{aligned}
$$

Let $X_{\Delta}$ be an intermediate variable; the process methods in the channel of $X_{\Delta}$ and $X_{\max }$ are the same; $X_{\Delta}$ and $X_{\max }$ are in different section; let $\xi$ be the estimated inversion position of each section. If the sign of received signal is positive and local signal is PN code itself, then $Z$ is used to express the correlation coefficient. If the sign of received signal is negative and local signal is the modulation result of PN code and square wave, then $F$ is used to express the correlation coefficient. $Z^{\prime}$ is used to express the correlation coefficient in other conditions.

If inversion position is in the first section and the sign is from positive to negative, (19) is established, and then it is derived as (20). Furthermore, (21) is established, due to $X_{\max }=X_{2}^{\prime}, X_{\min }=X_{2}$, and $X_{\Delta}=X_{1}^{\prime}$. Consider

$$
\begin{aligned}
X_{1} & =\xi \cdot Z+(L-\xi) \cdot F \\
X_{1}^{\prime} & =\xi \cdot F+(L-\xi) \cdot Z^{\prime} \\
X_{2} & =L \cdot F \\
X_{2}^{\prime} & =L \cdot Z^{\prime} \\
\xi & =\frac{\left(X_{2}^{\prime}-X_{1}^{\prime}\right) \cdot L}{X_{2}^{\prime}-X_{2}}, \\
\xi & =\frac{\left(X_{\max }-X_{\Delta}\right) \cdot L}{X_{\max }-X_{\min }} .
\end{aligned}
$$

In the same ways, if inversion position is in the first section and the sign is from negative to positive, (22) and (23) are established. If inversion position is in the second section and the sign is from positive to negative, (24) and (25) are established. If inversion position is in the second section and the sign is from negative to positive, (26) and (27) are established. Consider

$$
\begin{aligned}
X_{1} & =\xi \cdot F+(L-\xi) \cdot Z \\
X_{1}^{\prime} & =\xi \cdot Z^{\prime}+(L-\xi) \cdot F \\
X_{2} & =L \cdot Z \\
X_{2}^{\prime} & =L \cdot F \\
\xi & =\frac{\left(X_{2}-X_{1}\right) \cdot L}{X_{2}-X_{2}^{\prime}} \\
\xi & =\frac{\left(X_{\max }-X_{\Delta}\right) \cdot L}{X_{\max }-X_{\min }} \\
X_{1} & =L \cdot Z
\end{aligned}
$$

$$
\begin{aligned}
X_{1}^{\prime} & =L \cdot F \\
X_{2} & =\xi \cdot Z+(L-\xi) \cdot F \\
X_{2}^{\prime} & =\xi \cdot F+(L-\xi) \cdot Z^{\prime}, \\
\xi & =\frac{\left(X_{2}-X_{1}^{\prime}\right) \cdot L}{X_{1}-X_{1}^{\prime}}, \\
\xi & =\frac{\left(X_{\Delta}-X_{\min }\right) \cdot L}{X_{\max }-X_{\min }} \\
X_{1} & =L \cdot F \\
X_{1}^{\prime} & =L \cdot Z^{\prime} \\
X_{2} & =\xi \cdot F+(L-\xi) \cdot Z \\
X_{2}^{\prime} & =\xi \cdot Z^{\prime}+(L-\xi) \cdot F \\
\xi & =\frac{\left(X_{2}^{\prime}-X_{1}\right) \cdot L}{X_{1}^{\prime}-X_{1}}, \\
\xi & =\frac{\left(X_{\Delta}-X_{\min }\right) \cdot L}{X_{\max }-X_{\min }} .
\end{aligned}
$$

Finally, it can be derived from (21), (23), (25), and (27) that the message inversion position is

$$
\xi= \begin{cases}\frac{\left(X_{\max }-X_{\Delta}\right) \cdot L}{X_{\max }-X_{\min }}, & \left(X_{\max } \subset\left(X_{2}, X_{2}^{\prime}\right)\right) \\ L+\frac{\left(X_{\Delta}-X_{\min }\right) \cdot L}{X_{\max }-X_{\min }}, & \left(X_{\max } \subset\left(X_{1}, X_{1}^{\prime}\right)\right) .\end{cases}
$$

\section{Simulation and Analysis}

The new algorithm is simulated and analyzed based on MATLAB simulation platform. The basic parameters are as follows: message rate of $100 \mathrm{~Hz}, \mathrm{PN}$ code rate of $1 \mathrm{MHz}$, carrier frequency of $3 \mathrm{MHz}$, and sampling frequency of $12 \mathrm{MHz}$.

4.1. Algorithm Simulation. The simulation parameters in the first example are as follows: carrier frequency offset is $845 \mathrm{~Hz}$, PN code offset is $42 \mathrm{~ms}$, accumulation time is $10 \mathrm{~ms}$, SNR is $-12 \mathrm{~dB}$, and the message sign is always positive. The simulation parameters in the second example are as follows: carrier frequency offset is $1190 \mathrm{~Hz}, \mathrm{PN}$ code offset is $24 \mathrm{~ms}$, accumulation time is $10 \mathrm{~ms}$, signal to noise ratio is $-12 \mathrm{~dB}$, message sign is from negative to positive, and reversion position is $26.5 \mathrm{~ms}$.

The simulation results of search stage are shown in Figures 5 and 6 , and the proportion peak results are shown in Figures 7 and 8 . Figures 5 and 7 show that the proportion peak of the ninth channel is maximum value, thus preliminarily estimating carrier frequency as $800 \mathrm{~Hz}$ in the first example. Figures 6 and 8 show that the proportion peak of the 13th channel is maximum value, thus preliminarily estimating carrier frequency as $1200 \mathrm{~Hz}$ in the second example. 


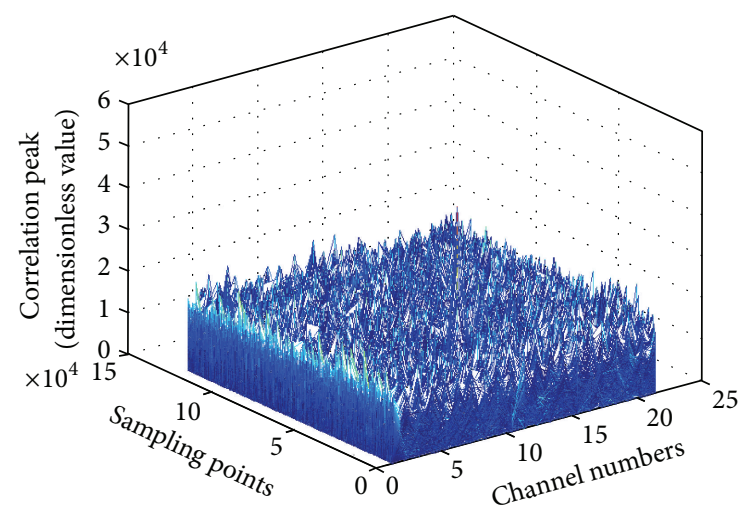

FIGURE 5: The simulation result of search stage in the first example.

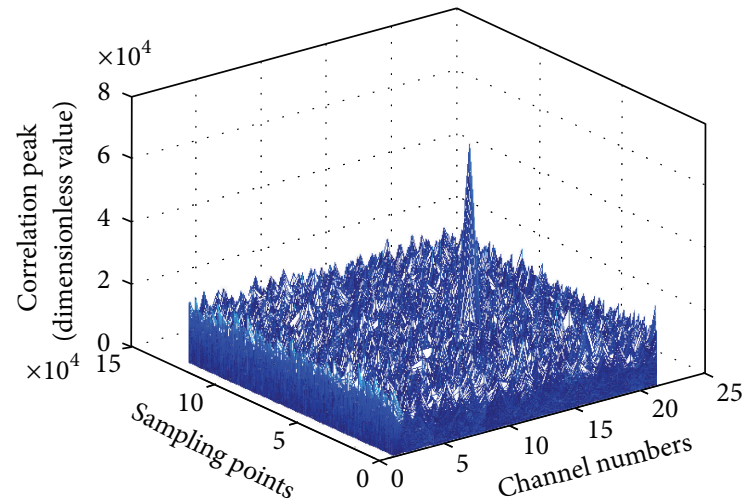

Figure 6: The simulation result of search stage in the second example.

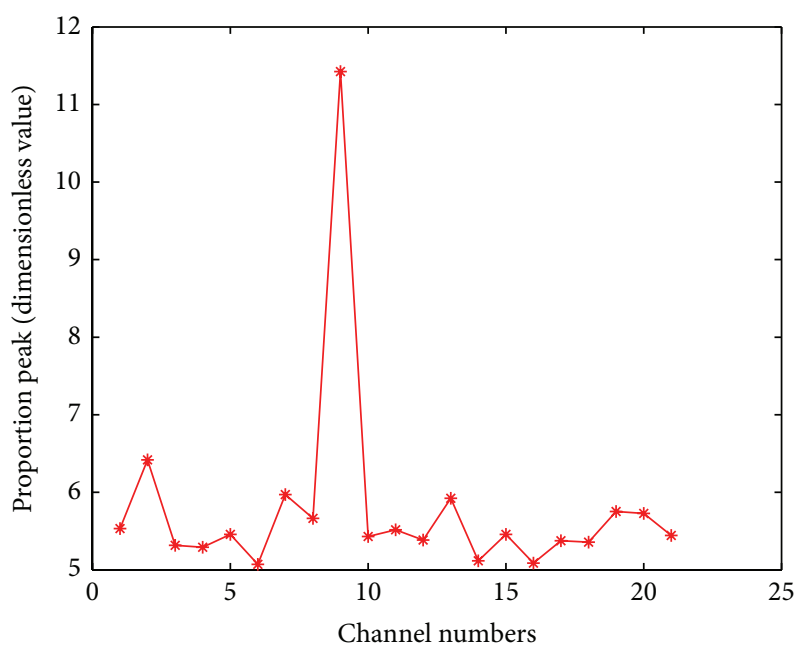

FIGURE 7: The proportion peak result of search stage in the first example.

In the coarse acquisition stage, the maximum proportion peak results of $I$ channel and Q channel are shown in Figures 9 and 10. Figure 9 shows that the maximum proportion peak of $Q$ channel is greater than $I$ channel; thus further estimated carrier frequency is $825 \mathrm{~Hz}$ in the first example. Figure 10 shows that the maximum proportion peak of $I$ channel is

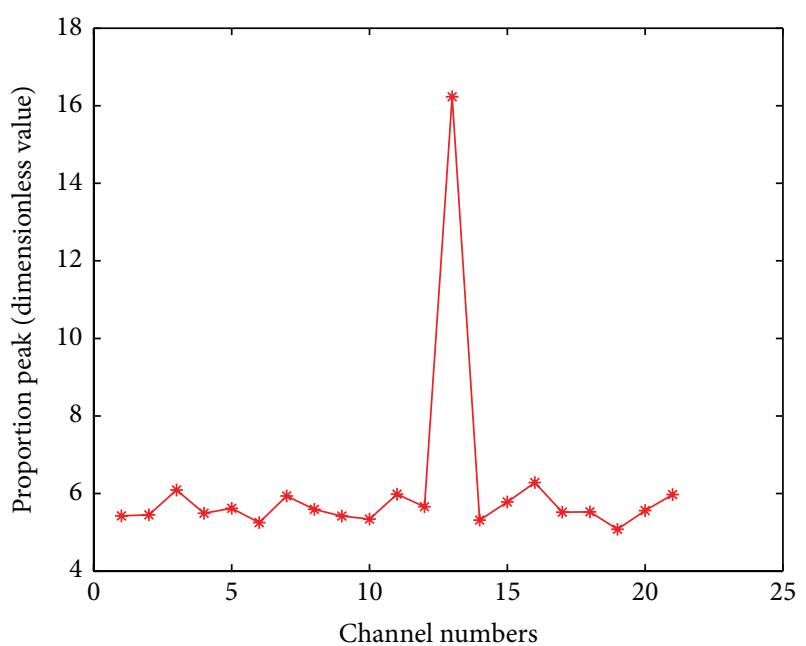

FIGURE 8: The proportion peak result of search stage in the second example.

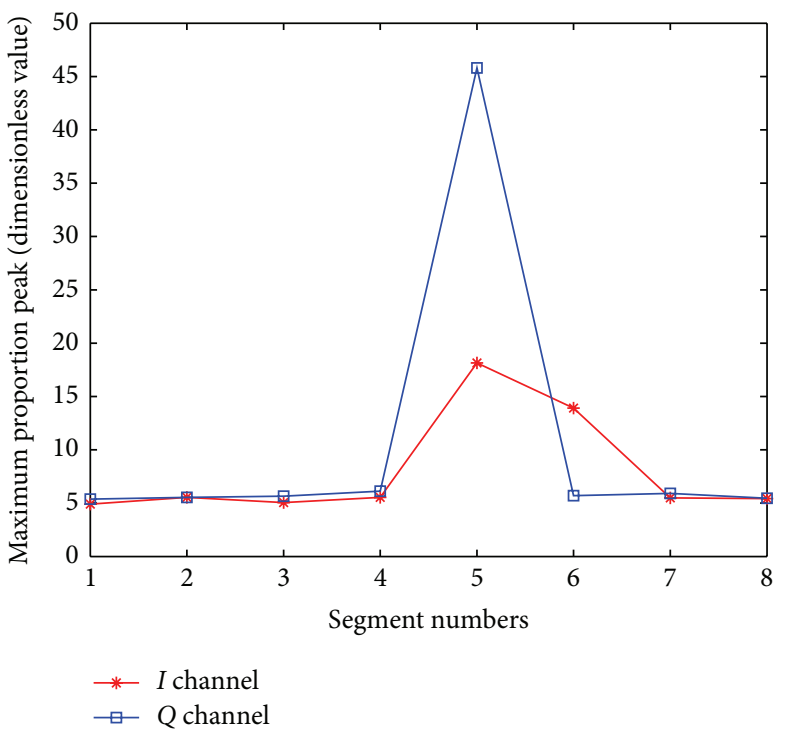

FIGURE 9: The maximum proportion peak of coarse acquisition stage in the first example.

greater than $Q$ channel, thus estimating carrier frequency as $1200 \mathrm{~Hz}$ in the second example.

At the same time, the PN code offset is estimated as $42 \mathrm{~ms}$ by the position of maximum proportion peak in the first example, and it is shown in Figure 11. The PN code offset is estimated as $24 \mathrm{~ms}$ in the second example, and it is shown in Figure 12. Four correlation peaks in the precise acquisition stage are used in (28); thus the message sign is estimated as positive in the first example. The message sign is estimated from negative to positive, and the estimated reversion position is $26.58 \mathrm{~ms}$ in the second example.

All simulation results of the first example show that the frequency error is $20 \mathrm{~Hz}$, the $\mathrm{PN}$ code phase error is 0 , and the message reversion error is 0 . The second example results 


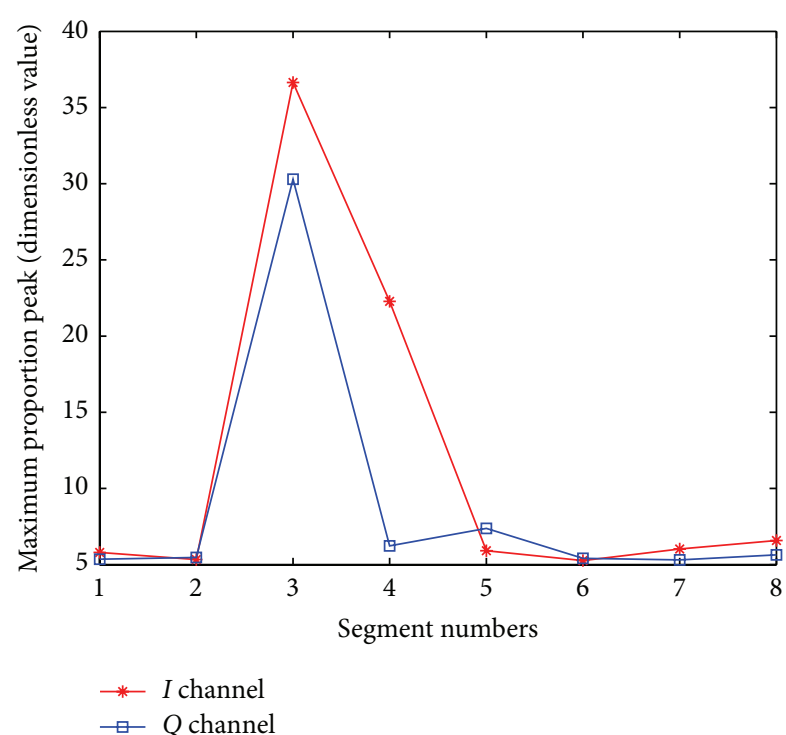

FIGURE 10: The maximum proportion peak of coarse acquisition stage in the second example.

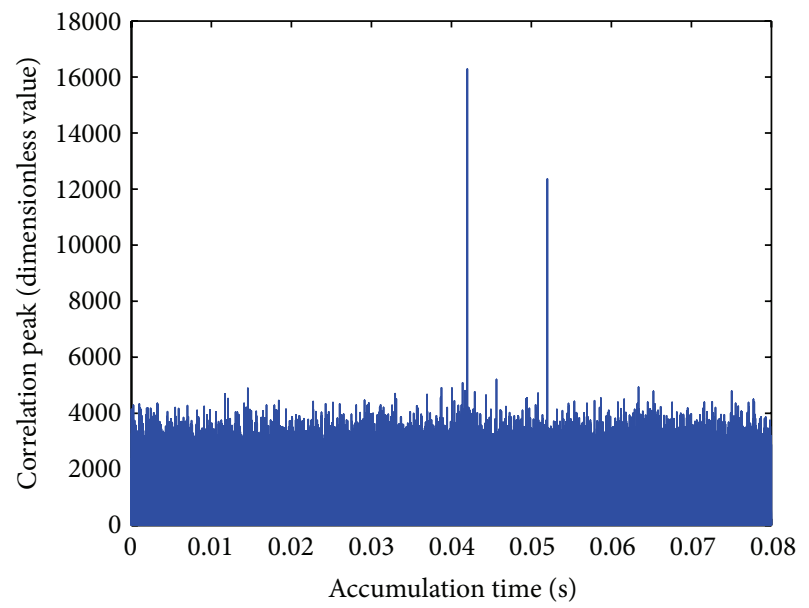

Figure 11: The final estimated PN code offset result in the first example.

show that the frequency error is $10 \mathrm{~Hz}$, the PN code phase error is 0 , and the message reversion error rate is $0.8 \%$.

4.2. Performance Analysis. This chapter aims to analyze this new algorithm adaptability and validity from diverse angles.

The effects of Doppler and message inversion on the search stage are not obvious because this stage is the initial acquisition stage. Therefore, the effects of the segment number selection and SNR are analyzed in this stage. The relationship between the segment number selection and the proportion peak is shown in Figure 13, which shows that the proportion peak decreases gradually along with the increase in segment number. The relationship between SNR and the proportion peak is shown in Figure 14, which shows that the proportion peak decreases gradually along with the decrease of SNR. Because the proportion peak will not meet

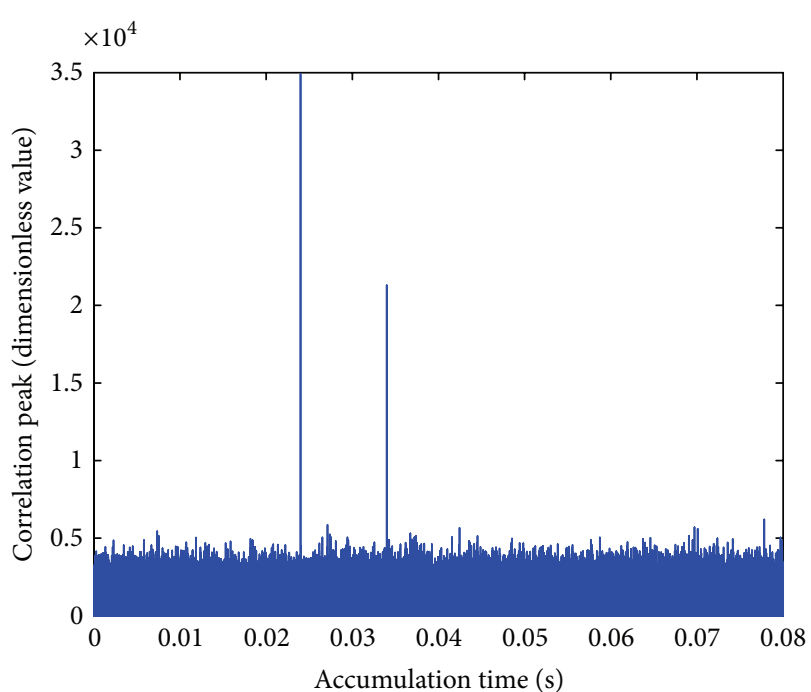

FIGURE 12: The final estimated PN code offset result in the second example.

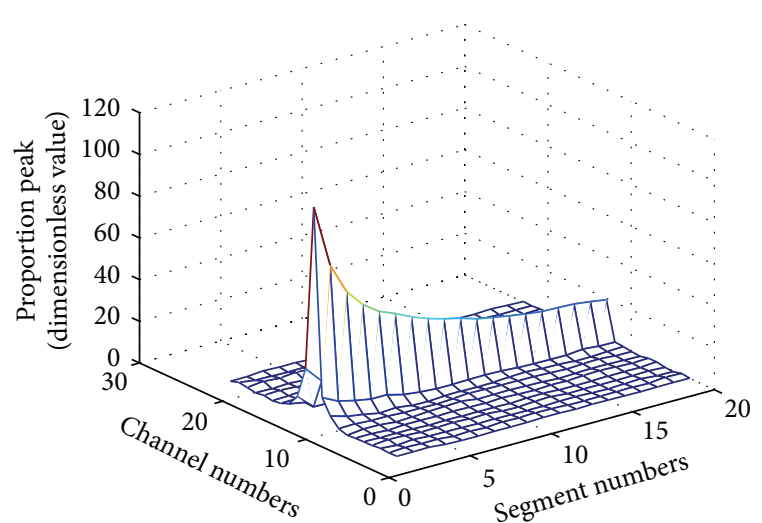

FIGURE 13: The relationship between the segment number and the proportion peak in search stage.

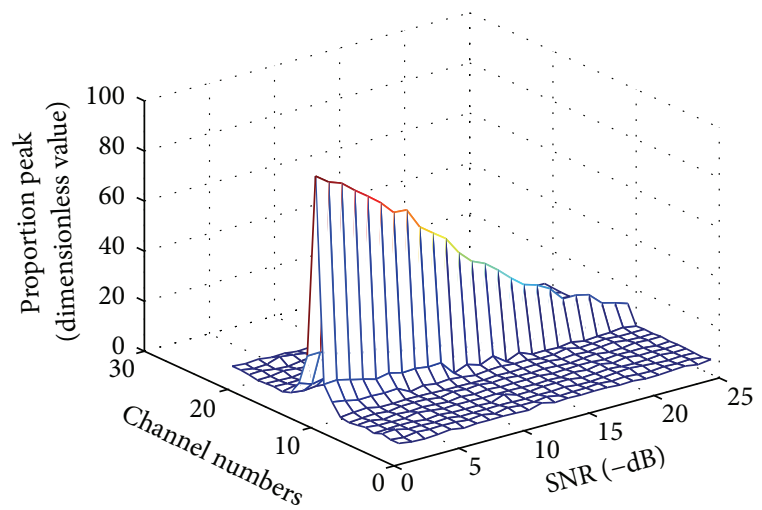

FIGURE 14: The relationship between the SNR and the proportion peak in search stage. 


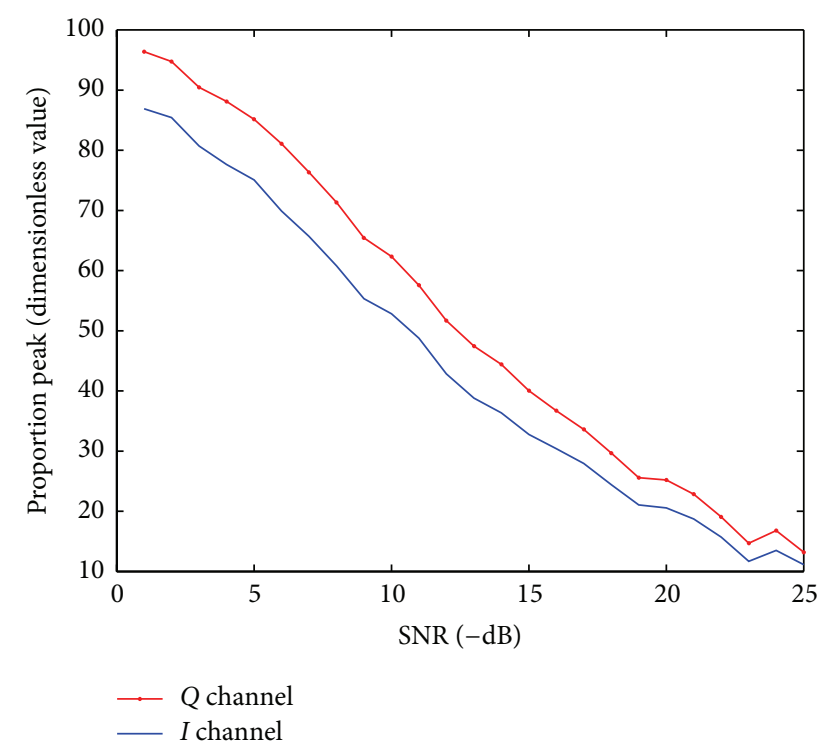

FIGURE 15: The relationship between the SNR and the proportion peak in coarse acquisition stage.

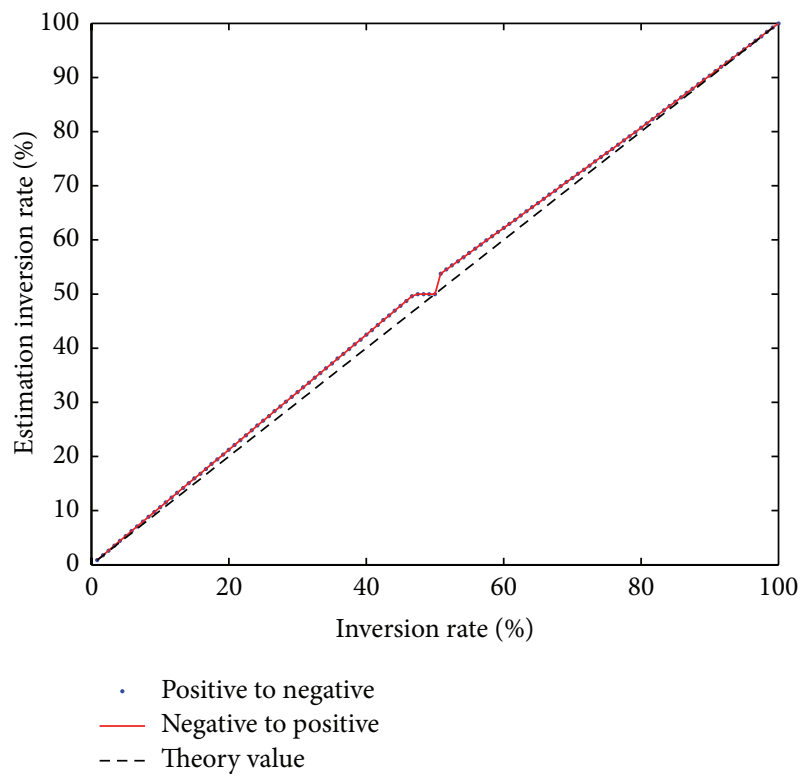

FIGURE 16: The estimation inversion rate result in precise acquisition stage.

the threshold requirement when SNR reaches $-25 \mathrm{~dB}$, the adaptation SNR of this algorithm is not less than $-25 \mathrm{~dB}$.

The effect of SNR in the coarse acquisition stage is obvious, and the relationship between SNR and the proportion peak is shown in Figure 15, which shows that the proportion peak decreases gradually along with the decrease of SNR. The proportion peak of $Q$ channel is always greater than $I$ channel, so the compensation frequency of $Q$ channel is more close to the real value in this condition.

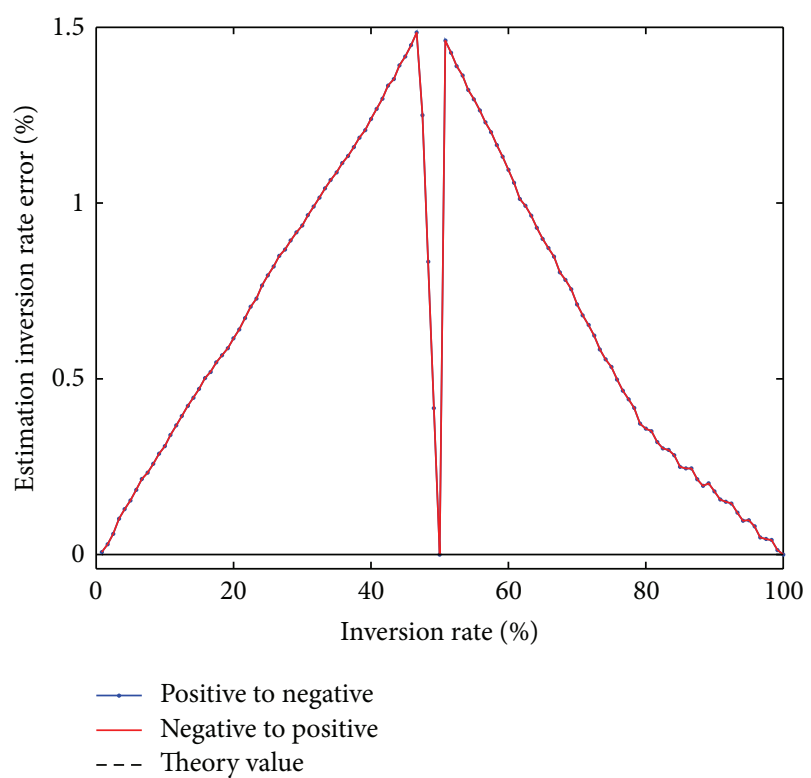

FIGURE 17: The estimation inversion rate error in precise acquisition stage.

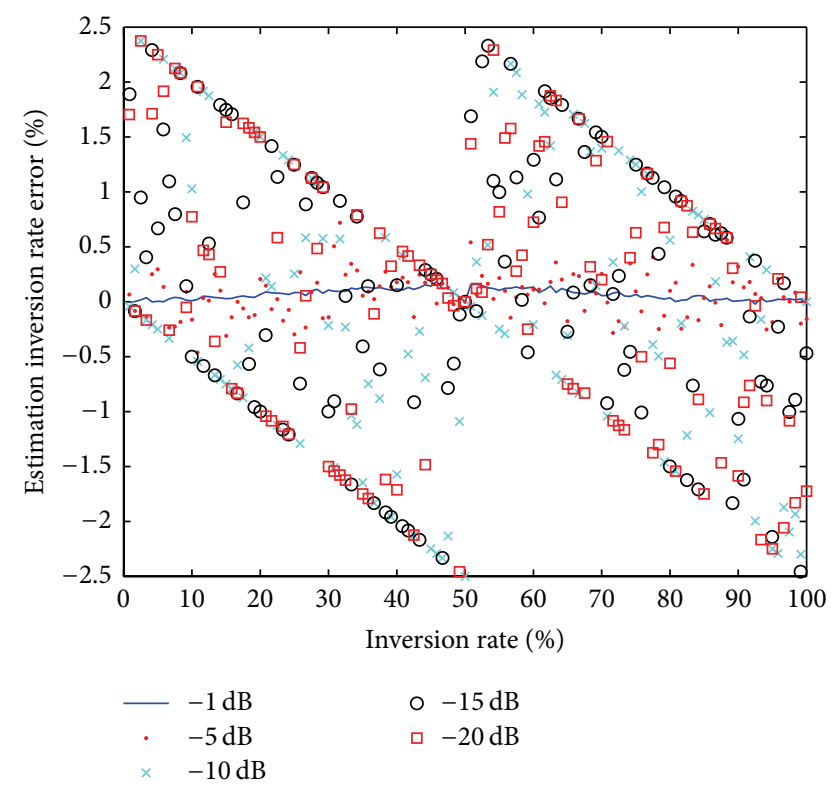

FIGURE 18: The relationship between estimation inversion rate error and SNR in precise acquisition stage.

The effects of Doppler, message inversion, and SNR on the precise acquisition stage are obvious because this stage is a precise stage. The estimation inversion rate result in precise acquisition stage is shown in Figure 16 under the conditions that Doppler error is zero and SNR is zero, and the estimation inversion rate error is shown in Figure 17. The results show that the estimation error is less than $1.5 \%$ and it can meet the acquisition requirement. Finally, the relationship between estimation inversion rate error and SNR is shown in Figure 18, which proves that the estimation error 
TABLE 1: The acquisition time of two algorithms with different search range.

\begin{tabular}{|c|c|c|c|c|c|c|c|c|c|c|}
\hline \multirow{2}{*}{ Algorithm } & \multicolumn{10}{|c|}{ Search range } \\
\hline & $100 \mathrm{~ms}$ & $200 \mathrm{~ms}$ & $300 \mathrm{~ms}$ & $400 \mathrm{~ms}$ & $500 \mathrm{~ms}$ & $600 \mathrm{~ms}$ & $700 \mathrm{~ms}$ & $800 \mathrm{~ms}$ & $900 \mathrm{~ms}$ & $1 \mathrm{~s}$ \\
\hline Two-channel algorithm & $69.15 \mathrm{~s}$ & $138.42 \mathrm{~s}$ & $206.18 \mathrm{~s}$ & $278.15 s$ & $346.98 \mathrm{~s}$ & $416.12 \mathrm{~s}$ & $485.81 \mathrm{~s}$ & $554.90 \mathrm{~s}$ & $624.12 \mathrm{~s}$ & $693.3 \mathrm{~s}$ \\
\hline New algorithm & $7.24 \mathrm{~s}$ & $14.46 \mathrm{~s}$ & $21.87 \mathrm{~s}$ & $29.43 \mathrm{~s}$ & $36.59 \mathrm{~s}$ & $43.7 \mathrm{~s}$ & $51.38 \mathrm{~s}$ & $58.52 \mathrm{~s}$ & $65.89 \mathrm{~s}$ & $73.59 \mathrm{~s}$ \\
\hline
\end{tabular}

TABLE 2: The estimation frequency error of two algorithms with different Doppler.

\begin{tabular}{|c|c|c|c|c|c|c|c|c|c|}
\hline \multirow{2}{*}{ Algorithm } & \multicolumn{9}{|c|}{ Doppler } \\
\hline & $10 \mathrm{~Hz}$ & $20 \mathrm{~Hz}$ & $30 \mathrm{~Hz}$ & $40 \mathrm{~Hz}$ & $50 \mathrm{~Hz}$ & $60 \mathrm{~Hz}$ & $70 \mathrm{~Hz}$ & $80 \mathrm{~Hz}$ & $90 \mathrm{~Hz}$ \\
\hline Two-channel algorithm & $10 \mathrm{~Hz}$ & $20 \mathrm{~Hz}$ & $30 \mathrm{~Hz}$ & $40 \mathrm{~Hz}$ & $50 \mathrm{~Hz}$ & $40 \mathrm{~Hz}$ & $30 \mathrm{~Hz}$ & $20 \mathrm{~Hz}$ & $10 \mathrm{~Hz}$ \\
\hline New algorithm & $10 \mathrm{~Hz}$ & $5 \mathrm{~Hz}$ & $5 \mathrm{~Hz}$ & $15 \mathrm{~Hz}$ & $25 \mathrm{~Hz}$ & $35 \mathrm{~Hz}$ & $30 \mathrm{~Hz}$ & $20 \mathrm{~Hz}$ & $10 \mathrm{~Hz}$ \\
\hline
\end{tabular}

TABLE 3: The estimation code phase error of two algorithms with different code phase.

\begin{tabular}{lcccccccccc}
\hline \multirow{2}{*}{ Algorithm } & \multicolumn{1}{c}{ Code phase } \\
& 1 chip & 2 chips & 3 chips & 4 chips & 5 chips & 6 chips & 7 chips & 8 chips & 9 chips & 10 chips \\
\hline Two-channel algorithm & $<1$ chip & $<1$ chip & $<1$ chip & $<1$ chip & $<1$ chip & $<1$ chip & $<1$ chip & $<1$ chip & $<1$ chip & $<1$ chip \\
New algorithm & $<1$ chip & $<1$ chip & $<1$ chip & $<1$ chip & $<1$ chip & $<1$ chip & $<1$ chip & $<1$ chip & $<1$ chip & $<1$ chip \\
\hline
\end{tabular}

TABLE 4: The estimation inversion error rate of two algorithms with different message inversion rate.

\begin{tabular}{|c|c|c|c|c|c|c|c|c|c|c|c|}
\hline \multirow{2}{*}{ Algorithm } & \multicolumn{11}{|c|}{ Inversion position } \\
\hline & $1 / 12$ & $2 / 12$ & $3 / 12$ & $4 / 12$ & $5 / 12$ & $6 / 12$ & $7 / 12$ & $8 / 12$ & $9 / 12$ & $10 / 12$ & $11 / 12$ \\
\hline Two-channel algorithm & $4.1 \%$ & $3.5 \%$ & $3 \%$ & $2.5 \%$ & $1.9 \%$ & $1.4 \%$ & $1.3 \%$ & $1.1 \%$ & $2 \%$ & $3 \%$ & $3.8 \%$ \\
\hline New algorithm & $0.3 \%$ & $0.5 \%$ & $0.8 \%$ & $1.1 \%$ & $1.3 \%$ & $0 \%$ & $1.2 \%$ & $0.9 \%$ & $0.6 \%$ & $0.3 \%$ & $0.1 \%$ \\
\hline
\end{tabular}

increases gradually along with the decrease of SNR, and the effect is irregular. From the previous results, it can be seen that this algorithm estimation inversion rate error is less than $2.5 \%$ in the condition of more than $-25 \mathrm{~dB}$.

4.3. Algorithm Comparison. In order to verify the superiority of the new algorithm, this algorithm is compared with twochannel algorithm on the premise of successful acquisition. The simulation parameters are as follows: carrier frequency offset is $845 \mathrm{~Hz}, \mathrm{PN}$ code offset is $42 \mathrm{~ms}$, accumulation time is $10 \mathrm{~ms}$, SNR is $-12 \mathrm{~dB}$, the message sign is from negative to positive, and the reversion position is $44.5 \mathrm{~ms}$.

With the change of search range, the acquisition time of the two algorithms is shown in Table 1, which shows that the processing speed of the new algorithm increases 9.5 times more than two-channel algorithm. With the change of Doppler, the estimation frequency error of the two algorithms is shown in Table 2, which shows that the new algorithm can obviously reduce the estimation frequency error 6 times less than the two-channel algorithm. Furthermore, with the change of code phase, the estimation code phase error of the two algorithms is shown in Table 3, which shows that the estimation code phase error of the two algorithms is not obvious. With the change of message inversion rate, the estimation inversion error rate of the two algorithms is shown in Table 4, which shows that the new algorithm can obviously reduce the estimation inversion error rate 38 times less than the two-channel algorithm.

\section{Conclusion}

In this paper, the principle and characteristics of TDDM spread spectrum signal are studied. At the same time, the fuzz problem caused by external factors and internal factors is analyzed, and the two-channel algorithm for TDDM signal is mainly studied. Aiming at the unique characteristics of TDDM signal, a fast acquisition algorithm is proposed to overcome the fuzz problems. The new algorithm is composed of three parts, which are search stage, coarse acquisition stage, and precise acquisition stage. In the search stage, the $I-Q$ frequency compensation processing is employed to overcome the frequency fuzz problem; the superposition processing of local code and square wave modulation is employed to overcome message reversal fuzz problem. Further, the search speed is enhanced by subsection processing. In the coarse acquisition stage, the signal phase and frequency are quickly determined by removing subsection processing and $I-Q$ frequency compensation processing. In the precise acquisition stage, the received signal and local code are divided into two sections, and four channel correlation results are combined to calculate message reversion position. Finally, the new algorithm is simulated. The simulation results 
show that the new algorithm can quickly estimate carrier frequency, code phase, and message inversion position. It can overcome the above fuzz problems, the adaptation SNR of this algorithm is not less than $-25 \mathrm{~dB}$, and this algorithm estimation inversion rate error is less than $2.5 \%$. In the same condition, the processing speed of the new algorithm is better than the traditional algorithm. And the estimation frequency error and inversion error of the new algorithm are obviously less than the traditional algorithm.

\section{Conflict of Interests}

The authors declare that there is no conflict of interests regarding the publication of this paper.

\section{Acknowledgments}

This work was supported by the Program for Liaoning Innovative Research Team in University (no. LT2011005), New Century Program for Excellent Talents of Ministry of Education of China (no. NCET-11-1013), Project of Science and Technology Department of Liaoning Province (no. 20121038), Project of Education Department of Liaoning Province (no. L2013085), and the Open Foundation of Key Laboratory of Shenyang Ligong University.

\section{References}

[1] Q. Zhang, X. Niu, H. Zhang, and C. Shi, "Algorithm improvement of the low-end GNSS/INS systems for land vehicles navigation," Mathematical Problems in Engineering, vol. 2013, Article ID 435286, 12 pages, 2013.

[2] X. Wang, B. Xu, and C. Luo, "An asynchronous communication system based on the hyperchaotic system of 6th-order cellular neural network," Optics Communications, vol. 285, no. 24, pp. 5401-5405, 2012.

[3] X. Wang and C. Luo, "Hybrid modulus-phase synchronization of hyperchaotic complex systems and its application to secure communication," International Journal of Nonlinear Sciences and Numerical Simulation, vol. 14, no. 7-8, pp. 533-542, 2013.

[4] J. K. Holmes and S. Raghavan, "A summary of the new GPS IIR$\mathrm{M}$ and IIF modernization signals," in Proceedings of the IEEE 60th Vehicular Technology Conference (VTC2004-Fall '04), pp. 4116-4126, Los Angeles, Calif, USA, September 2004.

[5] S. U. Qaisar and A. G. Dempster, "Assessment of the GPS L2C code structure for efficient signal acquisition," IEEE Transactions on Aerospace and Electronic Systems, vol. 48, no. 3, pp. 1889-1902, 2012.

[6] L. Simone, G. Fittipaldi, and I. A. Sanchez, "Fast acquisition techniques for very long PN codes for on-board secure TTC transponders," in Proceedings of the IEEE Military Communications Conference (MILCOM '11), pp. 1748-1753, Baltimore, Md, USA, November 2011.

[7] T. H. Ta, S. U. Qaisar, A. G. Dempster, and F. Dovis, "Partial differential postcorrelation processing for GPS L2C signal acquisition," IEEE Transactions on Aerospace and Electronic Systems, vol. 48, no. 2, pp. 1287-1305, 2012.

[8] D. V. Ratnam, A. Pasha, P. Swathi, and M. V. G. Rao, "Acquisition of GPS L1 signals using Cooley-tukey FFT algorithm," in Proceedings of the IEEE International Conference on Signal
Processing, Computing and Control (ISPCC '13), pp. 1-4, Solan, India, September 2013.

[9] S. R. Babu, P. Selvam, G. S. Rao, and J. Wang, "Optimization of GPS L1 acquisition using Radix-4 FFT," in Proceedings of the International Conference on Recent Trends in Information Technology (ICRTIT '11), pp. 875-879, Tamil Nadu, India, June 2011.

[10] F. Macchi-Gernot, M. G. Petovello, and G. Lachapelle, "Combined acquisition and tracking methods for GPS L1 C/A and L1C signals," International Journal of Navigation and Observation, vol. 2010, Article ID 190465, 19 pages, 2010.

[11] X. Li, L. Chen, B. Xu, and C. Zhang, "Study on the method of fast acquisition in LEO satellite DSSS communication system," in Proceedings of the IEEE 10th International Conference on Electronic Measurement and Instruments (ICEMI '11), pp. 325328, August 2011.

[12] W.-Y. Shin, M. Kim, G.-M. Hong, and S. Kim, "A fastacquisition PLL using split half-duty sampled feedforward loop filter," IEEE Transactions on Consumer Electronics, vol. 56, no. 3, pp. 1856-1859, 2010.

[13] K.-C. Kwon and D.-S. Shim, "Fast acquisition method for GPS L1/L2C software receivers," in Proceedings of the 11th International Conference on Control, Automation and Systems (ICCAS '11), pp. 1612-1615, October 2011.

[14] X.-M. Tang, S.-W. Yong, and F.-X. Wang, "Performance of XFAST in the presence of code Doppler," Journal on Communications, vol. 31, no. 8, pp. 54-59, 2010.

[15] X.-F. Wu, J.-A. Wang, C. Guo, S.-G. Hu, and Z.-F. Xi, "Adaptive direct acquisition algorithm based on XFAST," Journal of Central South University (Science and Technology), vol. 43, no. 11, pp. 4333-4338, 2012.

[16] X.-Y. Wang and J.-M. Song, "Synchronization of the fractional order hyperchaos Lorenz systems with activation feedback control," Communications in Nonlinear Science and Numerical Simulation, vol. 14, no. 8, pp. 3351-3357, 2009.

[17] X. Wang and Y. He, "Projective synchronization of fractional order chaotic system based on linear separation," Physics Letters A: General, Atomic and Solid State Physics, vol. 372, no. 4, pp. 435-441, 2008.

[18] Y.-X. Feng, B. Qian, and F. Liu, "Research on PN code synchronization algorithm for TDDM-based BOC modulation signal," Journal of Astronautics, vol. 32, no. 3, pp. 645-651, 2011.

[19] F. Liu and Y. Feng, "A long code acquisition algorithm on resolve time-frequency uncertainty problem," Acta Aeronautica et Astronautica Sinica, vol. 34, no. 8, pp. 1924-1933, 2013. 


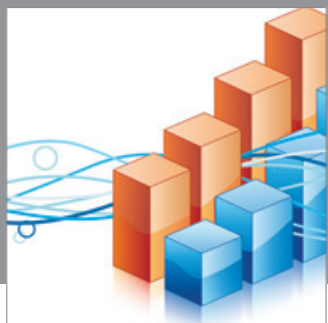

Advances in

Operations Research

mansans

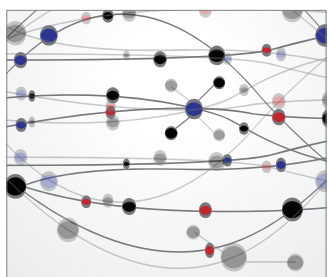

The Scientific World Journal
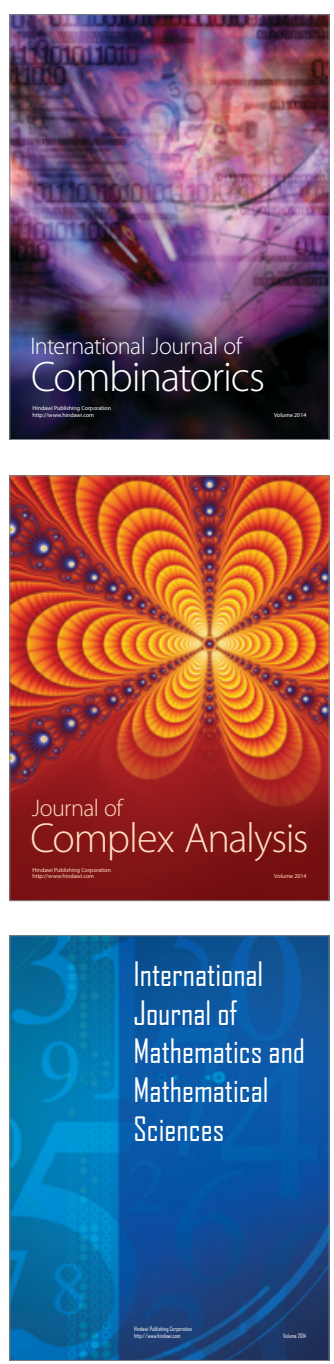
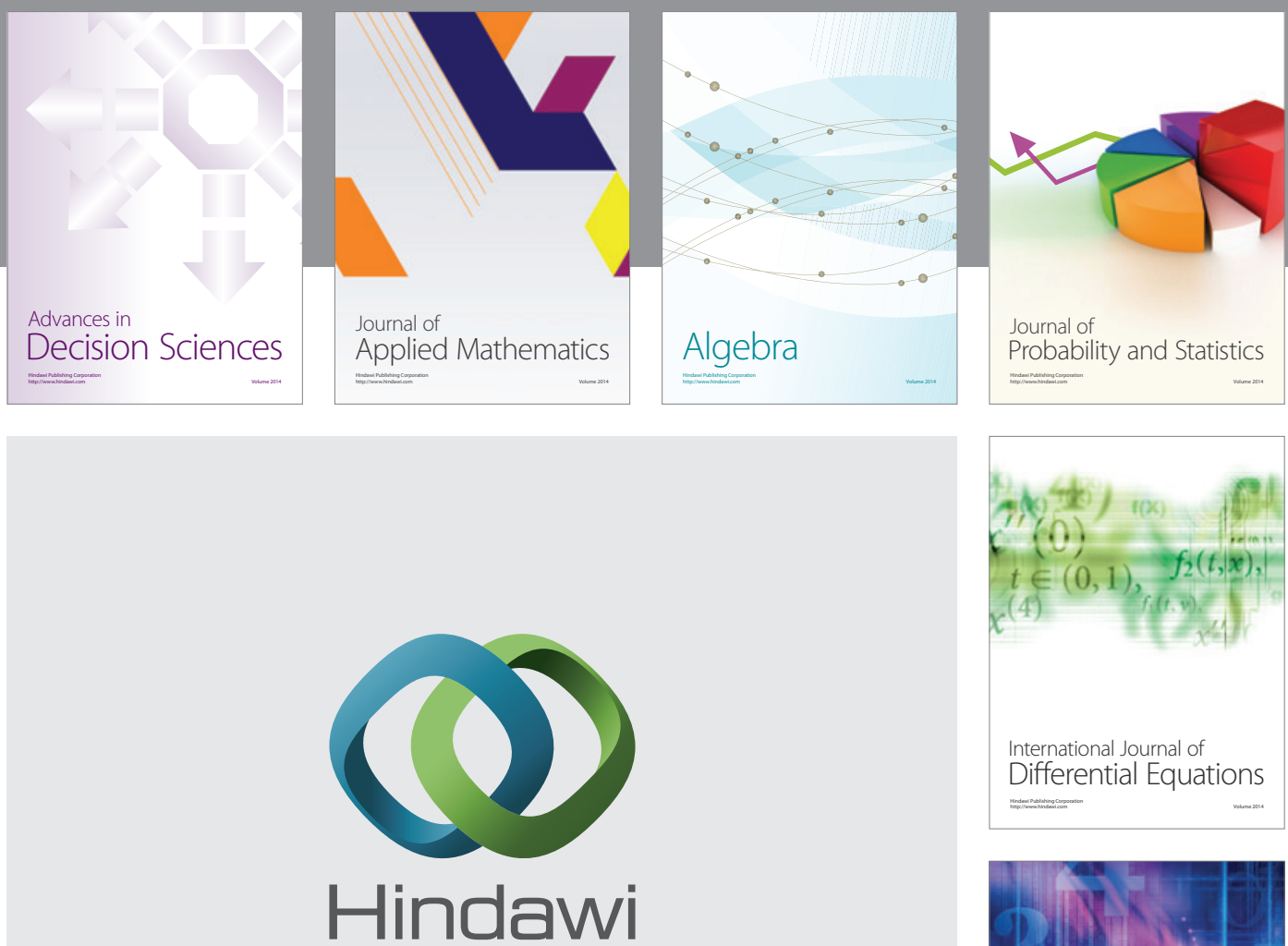

Submit your manuscripts at http://www.hindawi.com
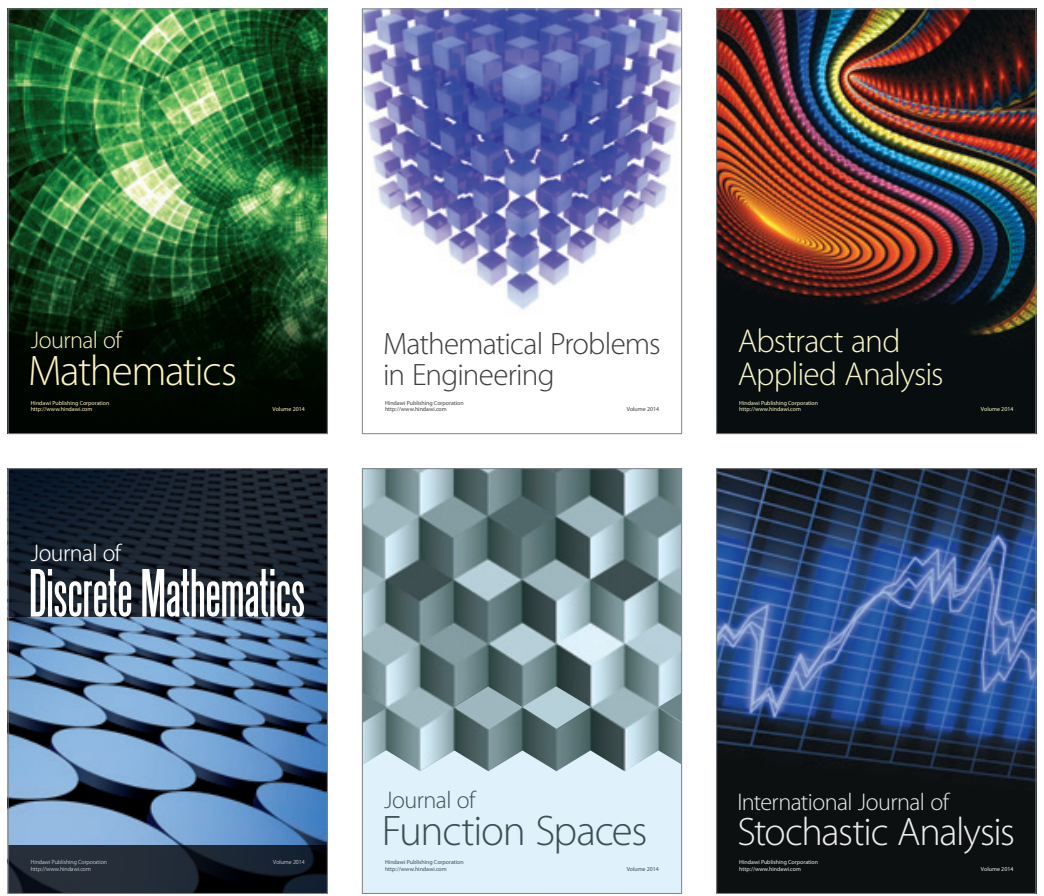

Journal of

Function Spaces

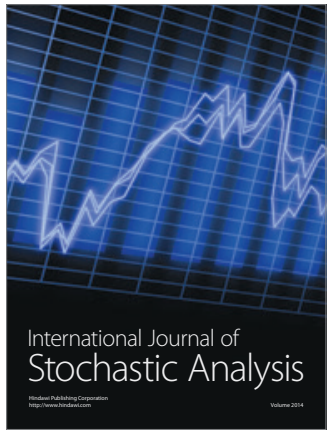

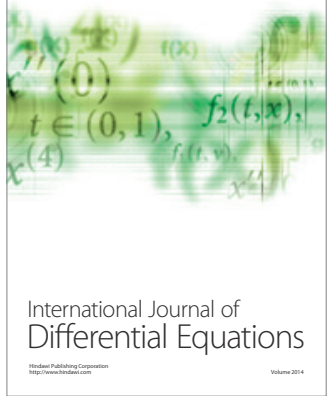
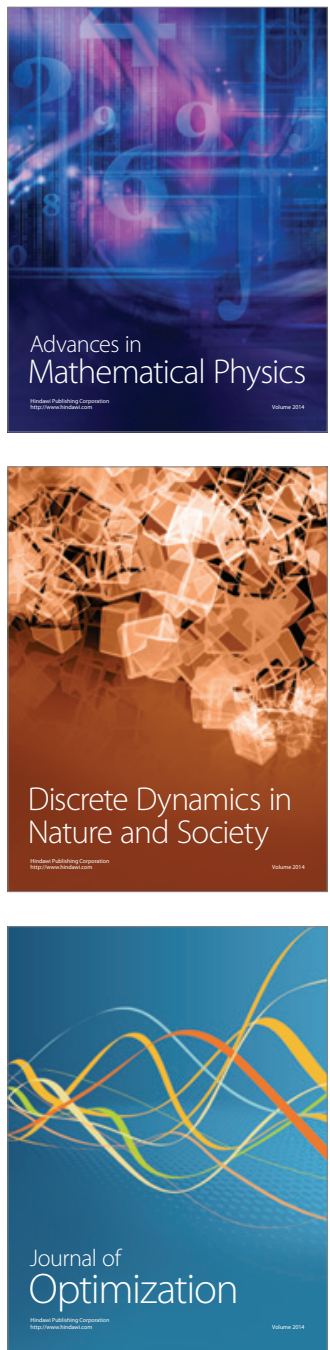